\title{
Distinct evolution of infection-enhancing and neutralizing epitopes in the spike protein of SARS- CoV-2 variants (from alpha to omicron) : a structural and molecular epidemiology study
}

\section{Patrick GUERIN}

OpenHealth

Nouara YAHI

Aix-Marseille University

Fodil AZZAZ

Aix-Marseille University

Henri CHAHINIAN

Aix-Marseille University

Jean-Marc SABATIER

Aix-Marseille University

Jacques FANTINI ( $\nabla$ jm.fantini@gmail.com )

Aix-Marseille University

\section{Research Article}

Keywords: SARS-CoV-2 variants, vaccine, facilitating antibodies, neutralizing antibodies, molecular epidemiology

Posted Date: December 13th, 2021

DOI: https://doi.org/10.21203/rs.3.rs-1054360/v2

License: (c) (i) This work is licensed under a Creative Commons Attribution 4.0 International License. Read Full License 


\title{
Distinct evolution of infection-enhancing and neutralizing epitopes in the spike protein of SARS-CoV-2 variants (from alpha to omicron) : a structural and molecular epidemiology study
}

\author{
Patrick Guérin'1, Nouara Yahi ${ }^{2}$, Fodil Azzaz ${ }^{2}$, Henri Chahinian², Jean-Marc Sabatier ${ }^{3}$ \& Jacques Fantini ${ }^{2}$ \\ ${ }^{1}$ OpenHealth - CP 130 - 56038 Vannes Cedex, France ; ${ }^{2}$ Aix-Marseille Univ, INSERM UMR_S 1072, \\ Marseille, France; ${ }^{3}$ Aix-Marseille Univ, CNRS, INP, Inst Neurophysiopathol, Marseille, France. \\ Correspondence: Jacques Fantini, Aix-Marseille University, France. \\ E-mail:jm.fantini@gmail.com
}

\begin{abstract}
Infection-enhancing antibodies may limit the efficiency of Covid-19 vaccines. We analyzed the evolution of neutralizing and facilitating epitopes in 1,860,489 SARS-CoV-2 genomes stored in the Los Alamos database from June to November 2021. The structural dynamics of these epitopes was determined by molecular modeling of the spike protein on a representative panel of SARS-CoV-2 variants. D614, which belongs to an antibodydependent-enhancement (ADE) epitope common to SARS-CoV-1 and SARS-CoV-2, has mutated to D614G in 2020, which could explain why ADE has not been detected following mass vaccination. A second epitope located in the N-terminal domain (NTD), specific of SARS-CoV-2, is highly conserved among most variants. In contrast, the neutralizing epitope of the NTD showed extensive variations in SARS-CoV-2 variants. The balance between facilitating and neutralizing antibodies is in favor of neutralization for the Wuhan strain, alpha and beta variants, but not for gamma, delta, lambda, and mu. The recently emerging omicron variant is atypic as its mutational profiles affects both neutralization and ADE epitopes. Overall, our data reveal that the evolution of SARS-CoV-2 has dramatically affected the ADE/neutralization balance. Future vaccines should consider these findings to design new formulations adapted to SARS-CoV-2 variants and lacking ADE epitopes in the spike protein.
\end{abstract}

Key words: SARS-CoV-2 variants; vaccine; facilitating antibodies; neutralizing antibodies; molecular epidemiology. 


\section{Introduction}

Cytotoxic T-cells and neutralizing antibodies play a key role in the control of viral infections, especially in the case of respiratory viruses $[1,2]$. However, virus-specific antibodies can also promote pathology, a phenomenon referred to as antibody-dependent enhancement (ADE) [3]. ADE of virus infection is generally due to virus-specific antibodies that enhance the entry of virus into host cells, and in some cases, virus replication in monocytes, dendritic cells and macrophages through antibody binding to Fc $\gamma$ receptors [4]. In addition, alternative mechanisms of ADE involving the complement component $\mathrm{C} 1 \mathrm{q}$ have been reported [5]. ADE has been observed in two typical situations: i) reinfection with a virus variant after primary infection with a different strain [6] or a cross-reactive virus [7], and ii) as the result of viral infection in vaccinated people [8]. The ADE phenomenon was initially discovered in flaviviruses in the late 1960's [9] and experimentally demonstrated in the early 1970's [10]. It concerns a broad range of viruses including dengue [11], Ebola [12], Zika [13], HIV [14], influenza [15], and various animal and human coronaviruses [16].

As early as in June 2020, at a time when Covid-19 vaccines had just entered clinical evaluation, Akiko Iwasaki and Yexin Yang from Yale University School of Medicine alerted that " $A D E$ should be given full consideration in the safety evaluation of emerging candidate vaccines for SARS-CoV-2" [17]. A similar warning on vaccine safety due to potential risks of ADE was independently published by Shibo Jiang [18]. In contrast, several authors considered the risk to be null or minimal in the case of SARS-CoV-2 [19] [20] [21] [22].

However, several pieces of evidence strongly argue in favor of an ADE issue for SARS-CoV2. i) ADE has been reported for animal coronaviruses such as feline infectious peritonitis virus [23]. In the most dramatic cases, kittens previously vaccinated with a recombinant virus containing the spike protein gene succumbed of early death after a coronavirus challenge [24]. ii) $\mathrm{ADE}$ epitopes were characterized in the spike protein of this feline coronavirus [25]. iii) ADE epitopes have also been found in human coronaviruses related to SARS-CoV-2, i.e. SARS-CoV-1 [26] and MERS-CoV [27] [28]. The case of SARS-CoV-1 is particularly interesting since its spike protein displays a linear ADE epitope, 597-LYQDVNC-603 (recognized by the monoclonal antibody 43-3-14) [26] that is fully conserved in the SARSCoV-2 spike protein sequence used for mRNA Covid-19 vaccines. iv) ADE antibodies directed against the N-terminal domain (NTD) of the spike protein have been detected and characterized in convalescent Covid-19 patients [29] [30]. v) ADE antibodies are suspected to 
be particularly efficient in vaccinated Covid-19 patients infected with the delta variant [31] [32]. In this context, we recently reported that facilitating anti-spike antibodies targeting the NTD have a higher affinity for the delta variant than for the initial Wuhan strain. We also reported that the main neutralizing epitope of the NTD is almost lost in $\delta$ variants [31]. This finding is of critical importance since ADE infection of coronaviruses is known to be induced by the presence of sub-neutralizing levels of anti-spike antibodies [33]. Overall, our data suggested that the balance between neutralizing and facilitating antibodies may greatly differ according to the virus strain.

In the present study, we analyzed a panel of representative SARS-CoV-2 variants including alpha, beta, gamma, delta, lambda, mu as well as the most recent South-Africa strains C.1.2 (with no attributed Greek letter at the time of submission) and omicron. We used multiple amino acid sequence alignment methods combined with structural and molecular modeling approaches to determine the variability of ADE and neutralizing epitopes and the impact of this variability on antibody-spike protein interactions. Our main objectives were i) to decipher the evolution of neutralizing and facilitating epitopes since the beginning of the Covid-19 pandemic, and ii) to predict for each SARS-CoV-2 variant which way the balance between neutralization and facilitation is tipping.

\section{Methods}

Molecular modeling studies were performed with Hyperchem (http://www.hyper.com) and Deep View/Swiss-Pdb viewer (https://spdbv.vital-it.ch) programs, as described in previous studies [34] [35] [36] [37]. The energy of interaction $(\Delta G)$ of each antibody-spike protein complex was calculated with Molegro Molecular Viewer (http://molexus.io/molegromolecular-viewer). The cluster of gangliosides GM1 in a typical lipid raft organization was generated as described previously from CHARMM-GUI Glycolipid Modeler [38] and submitted to several minimization steps with the Polak-Ribière algorithm [39].

\section{Results}

\section{Description of two distinct ADE epitopes in SARS-CoV-2 spike protein}

The mutational patterns and geographic origins of the SARS-CoV-2 variants analyzed in this study are summarized in Table 1. All variants have a dual nomenclature (lineage and Greek letter) except for C.1.2 which, at the date of submission of this article, had no Greek letter 
attributed. Our analysis is focused on the NTD and on the rod-like domains of the spike protein. Other $\mathrm{ADE}$ and neutralization epitopes do exist in the RBD, but during the complex process of viral adhesion to target cells, this domain is involved at later step [36] [37]. Clearly, the NTD is key to understand how SARS-CoV-2 initially interacts with the plasma membrane of host cells.

The first ADE epitope studied is the 611-617 motif with the original amino acid sequence LYQDVNC recognized by the 43-3-14 antibody [26]. This ADE epitope is common to human coronaviruses SARS-CoV-1 and SARS CoV-2. Interestingly, this epitope is centered on position 614 which is an aspartic acid residue in the original Wuhan strain but has rapidly evolved to the ultra-dominant D614G during the first months of 2020 [40]. The localization of this epitope on the spike protein (Wuhan strain) is shown in Figure 1A (epitope colored in yellow, except for D614 highlighted in red). It is well exposed on the protein surface so that it can be recognized by facilitating antibodies generated during previous coronavirus infections in humans, especially in geographic areas previously exposed to SARS-CoV-1. The second ADE epitope targeted by facilitating antibodies is divided in two parts (both colored in blue in Figure 1A): one in the NTD (27-32, 64-69 and 211-218 segments) and the other one in the rod-like domain (600-607, 674-677 and 689-691 segments) of the spike protein. Antibodies directed against this epitope have been detected in sera from convalescent Covid-19 patients[30]. Although the two parts of this ADE epitope seem to be spatially distant, both are close to a flexible 20-amino acid residue loop (621-640) that is unresolved in PDB files but was added by molecular modeling in the structures shown in Figure 1. It is interesting to note that this loop (highlighted in green) is ideally located to connect the NTD and the RBD, but also to provide a conformational link between both ADE epitopes (Figure 1B).

Once the NTD is bound to the cell membrane of the host cell, a conformational change unmasks the RBD which becomes available for a functional interaction with a viral receptor, chiefly ACE2 [37]. This spatial reorganization leads to the open, fusion-compatible conformation of the trimeric spike protein [41]. In the Wuhan strain, the closed conformation of the trimer [42] is stabilized by a hydrogen bond between D614 of one subunit and T859 of its neighbor (respectively chains B and C in Figure 2A). The global spreading of the pandemic during the first months of 2020 has been associated with the breakthrough of the first SARS-CoV-2 variant with a unique mutation in spike protein, D614G. As shown in Figure 2B, this mutation induces the loss of the hydrogen bond that stabilized the closed conformation. Thus, we analyzed the status of this hydrogen bond in the complex between the 
facilitating 1052 antibody and the spike protein trimer. As shown in Figure 2C, the antibody has a long range conformational effect on both D614 and T859, which renders impossible the formation of this hydrogen bond. It is likely that the 621-640 loop, which conformationally connects the 1052 and the 611-617 epitopes, mediates this distal effect. In this respect, it is interesting to note that this facilitation can be induced by two distinct mechanisms: i) the replacement of aspartic acid by a glycine at position 614 (D614G mutation), or ii) binding of the ADE antibody 1052 to the original Wuhan spike protein displaying an aspartic acid (D614) at this position.

\section{Analysis of amino acid sequence variations in ADE and neutralizing epitopes during the global spreading of the Covid-19 pandemic}

Then, we analyzed the evolution of the amino acid sequence of ADE epitopes among SARSCoV-2 variants (Figure 3). The 611-617 epitope (lower left panel), which is common to SARS-CoV-1 and SARS-CoV-2, has a unique signature in all variants, i.e. the D614G mutation. As position 614 is central to the epitope, this epitope is probably no longer recognized by ADE antibodies generated by previous coronavirus infections in humans. The second ADE epitope is formed by several distinct areas in the NTD and in the rod-like regions of the spike protein (Figure 3, upper panel). In the NTD, the epitope is divided in three linear segments that represent ca. $80 \%$ of the total energy of interaction of the 1052 antibody-NTD complex (as calculated from PDB: 7LAB): 27-32, 64-69 and 211-219 (accounting respectively for 12,19 and $51 \%$ of the energy of interaction). The complex is further stabilized by auxiliary contacts with the rod-like region of the spike protein (chiefly 600-607, 674-677 and 689-691). Overall, the whole epitope appeared to be extremely well conserved, except at two amino acid residues positions: H69 and D215. Indeed, a deletion $(\Delta H 69)$ is found in the alpha variant, and D215 is mutated in D215G in the beta and the more recent C1.2 variants (Figure 3, upper panel). Surprisingly, the recently emerging omicron variant does not seem to follow this general rule as its ADE epitope is heavily affected by a combination of single point mutations (A67V, L212I), two deletions $(\Delta \mathrm{H} 69, \Delta \mathrm{N} 211)$, and a 3aminoi acid insertion (between R214 and D-215).

In marked contrast with the conservation of the $1052 \mathrm{ADE}$ epitope in most variants, the main neutralizing epitope of the NTD showed extensive amino acid sequence variations (Figure 3, lower right panel). The changes included deletions, insertions and single point mutations that 
are distributed among two key regions, 144-158 (N3 loop) and 242-249 (N5 loop) that constitute the three-dimensional site recognized by the neutralizing 4A8 antibody [43]. The localization of the neutralization epitope of the NTD at the virus/host cell interface is consistent with this high variability as it is submitted to a strong pressure of selection for SARS-CoV-2 variants. Conversely, the ADE epitope, which is on the lateral side of the NTD, is not facing the plasma membrane of the host cell and for this reason is not subjected to such a high selective pressure.

The frequency of amino acid sequence variations of the ADE and neutralizing epitopes was analyzed by specific queries of the Los Alamos database over the last six-month period (2021-06-01 to 2021-11-27) (Table 2). All the epitopes listed in Figure 3 were analyzed in $1,860,489$ genomes. The ADE epitope of the NTD is highly conserved ( $>98 \%$ for all segments) except for the 64-69 motif at position H69 (variation of 5.46\% with 1 mutation), mostly reflecting the alpha variant [44]. The ADE epitope 611-617 displays 1 mutation in $98.70 \%$ of cases, consistent with the worldwide dominance of the D614G mutant [45]. The situation of the neutralization epitope of the NTD is by far more complex, in particular for the 144-158 segment which shows high amino acid sequence variability (frequency of the Wuhan sequence $<0.05 \%$ ). Remarkably, $92.04 \%$ of the sequences have 2 mutations and some viruses with 3, 4 and even 5 mutations are currently detected. The second linear segment (242-249) is more conserved (99.22\% of sequences are identical to the Wuhan strain), but viruses with up to 4 mutations have been characterized. Interestingly, the amino acid variations of the neutralization epitope are concentrated on positions that are associated with the variants analyzed in the present study: Y144, E156, F157 and R158, in the N3 loop, R246 in the N5 loop (Figure 3).

\section{Estimating the risks of the facilitation phenomenon depending on the variant concerned: a molecular modeling approach}

Finally, we used molecular modeling approaches to determine how mutations in ADE epitopes could impair the binding of facilitating antibodies. In our analysis of ADE epitopes in SARS-CoV-2 variants (Figure 3), we detected two essential mutations that can potentially suppress the facilitation phenomenon: $\Delta \mathrm{H} 69$ and D215G. Thus, we studied the localization of H69 and D215 in the molecular complex between ADE antibody 1052 and the spike protein (Figure 4A, left panel). Both H69 and D615 appeared critical for the 1052 antibody binding 
site on the NTD of the spike protein. These positions are fully conserved in the gamma, delta, mu and lambda SARS-CoV-2 variants, which are still recognized by the ADE antibody 1052 . An illustration of the efficiency of this antibody to facilitate the infection by the $\delta$ variant is shown in Figure 4A (right panel). The plasma membrane of the host cell is represented by a cluster of gangliosides GM1 to figure the lipid raft that acts as a landing platform for the NTD [36]. In line with previous data from our group [31], once the 1052 antibody is bound to the NTD of the delta spike protein, a global conformation change involving both the NTD and the antibody allows the formation of a highly energetic trimolecular complex (antibody-NTDlipid raft) with an obvious geometric complementarity of all partners. Then, we compared the structure of the delta variant NTD with the mu, lambda, and C.1.2 variants (Figure 4B). Except for C.1.2 which displays a D215G mutation, and the highly divergent omicron (Figure 3) all other variants have both H69 and D215 accessible on the NTD surface.

In line with these data, the energy of interaction of the C.1.2 variant spike protein with the 1052 antibody was less than half the value calculated for the Wuhan strain $\left(-229 \mathrm{~kJ} \cdot \mathrm{mol}^{-1}\right)$, whereas it reached $-246 \mathrm{~kJ}_{\mathrm{mol}}{ }^{-1}$ for the delta variant [31], $-236 \mathrm{~kJ} \mathrm{~mol}^{-1}$ for mu and -228 $\mathrm{kJ} \mathrm{mol}^{-1}$ for lambda variants. Thus, the conservation of H69 and D215 (in gamma, delta, mu and lambda variants) is critical for virus infectivity as it favors the ADE phenomenon by allowing an optimal binding of the 1052 antibody to the spike protein. In contrast, the ADE epitope is affected as soon as at least one of these positions is mutated (as it the case for alpha, beta, C.1.2 and omicron variants).

\section{Discussion}

Vaccine strategies against viral diseases are confronted to the risk of antibody facilitation (ADE), especially when the strain used for the immunization protocol is distinct from circulating viruses [46]. In the past, $\mathrm{ADE}$ has been evidenced for a broad range of human RNA viruses including HIV, influenza, filoviruses, and coronaviruses [4]. Although ADE antibodies have been consistently characterized in the serum from Covid-19 convalescent patients [29] [30], the risk of ADE linked to vaccination with spike protein-based vectors (either mRNA or adenovirus) has not been considered as critical. As a matter of fact, it has been generally assumed that ADE antibodies exhibited SARS-CoV-2 infection enhancement in vitro but not in vivo [30]. However, a potential caveat of these studies is that SARS-CoV-2 variants have not been specifically assessed. Moreover, surprising higher incidence rates in 
vaccinated vs. unvaccinated individuals in the 0-14 days after the first dose were recently reported in long-term care facility residents and health-care workers, which resulted in significant negative vaccine efficiency estimates of $-37 \%$ and $-113 \%$, respectively [47]. To which extent this apparent enhancement of SARS-CoV-2 infection is due (or not due) to an imbalance between vaccine-induced (and/or pre-existing) neutralizing and facilitating antibodies warrants further investigation. Moreover, a recent report revealed that there is no clear relationship between the percentage of fully vaccinated individuals and new Covid-19 cases in 68 countries including Israel, a pioneer in mass vaccination against SARS-CoV-2 [48]. Taken together, these observations suggested that ADE, or more specifically the $\mathrm{ADE} /$ neutralization balance, could pose a problem for Covid-19 vaccine strategies, especially during the outbreak of SARS-CoV-2 variants. Finally, it is worth noting that ADE has been suspected to increase the severity of Covid-19 symptoms in selected geographic areas [49]. The objective of the present study was thus to assess the potential risk of ADE in vaccinated individuals challenged with SARS-CoV-2 variants. To this end, we studied the amino acid sequence variability of ADE and neutralizing epitopes in the NTD and rod-like regions of the spike protein. Then we used our target-based molecular modeling strategy to interpret these data at the level of the three-dimensional structure of the spike proteins.

We focused our attention on two distinct ADE epitopes: one linear epitope common to SARSCoV-1 and SARS-CoV-2 (611-617 in the rod-like region of the spike protein, recognized by the 43-3-14 antibody) [26] and a complex three-dimensional NTD epitope (recognized by the 1052 antibody) [30].

Both epitopes are present on the spike protein generated by mRNA vaccines as the original formulas are based on the Wuhan strain [50]. Therefore, it is of high importance to determine whether these epitopes are still expressed and accessible on SARS-CoV-2 variants. The 611617 epitope has probably escaped facilitating antibodies because the D614G variant has rapidly replaced the original strain [45]. Although in the initial study of the D614G mutation the authors mentioned the presence of D614 in a conserved ADE epitope, they did not comment further this important issue [40]. Our modeling approaches revealed a common molecular mechanism leading to enhanced infectivity for the D614G variant and for ADE antibodies with the Wuhan strain (Figure 2). In both cases, the loss of a stabilizing hydrogen bond between amino acid residues 614 and 859 of two vicinal spike protein chains relaxes the trimer and facilitates the conformational change that unmasks the RBD. A major outcome of our study is the identification of the 621-640 loop, which is missing in PDB files, as the 
conformational transmitter that allows the 1052 antibody to induce distant effects on amino acid residue 614. In this respect, the enhancement of infection provided by this ADE antibody involves two distinct $\mathrm{Fc} \gamma$-independent mechanisms: a long range conformational effect and a stabilization of the NTD bound to a lipid raft [31].

From an epidemiologic point of view, we can propose a scenario according to which the first cases of SARS-CoV-2 infections in China could have been facilitated by ADE antibodies directed against the 611-617 epitope in individuals previously infected by SARS-CoV-1 or similar coronaviruses. This notion is supported by the recent demonstration that nonneutralizing antibodies directed against SARS-CoV-1 and recognizing the SARS-CoV-2 spike protein may persist for at least 15 years [51]. Then the global extension of SARS-CoV-2 has probably levied this constraint by selecting the D614G variant in SARS-CoV-1 free populations. This scenario is consistent with the rapid raise and long-term maintenance of D614G worldwide. It is also consistent with the discrepancy between the severity of Covid-19 cases observed in the Hubei province of China and those occurring elsewhere in the world at the beginning of the pandemic [49]. Moreover, it explains why ADE has not been detected during the first months following mass vaccination, since ADE antibodies directed against the 611-617 epitope are no longer active on D614G variants. The observation that anti-SARSCoV-1 antibodies isolated from a convalescent patient could enhance virus infection mediated by civet virus spike proteins [52] also supports this notion. Retrospectively, it is important to note the statement of Helen Pearson in a Nature editorial commenting these data in 2005: " $a$ jab against one strain might even aggravate an infection with SARS virus from civets or another species" [53].

After the first wave of D614G, several other SARS-CoV-2 variants have emerged until the rise of the delta variant which is now by far the most common strain worldwide. Indeed, key variations in the ADE epitope at positions 69 and 215 have probably protected patients infected with alpha or beta strains from the ADE risk (Figure 3). Nevertheless, these variants also showed significant variability of the neutralizing epitope, which could have decreased vaccine efficiency [54]. The situation is more dramatic for the delta variant. Indeed, several studies converged to alert on the potential risk of ADE when a delta SARS-CoV-2 variant infects a vaccinated individual [31] [32]. Our study confirms this possibility and further extends it to other circulating variants, including lambda and $\mathrm{mu}$, for which the neutralization/facilitation balance is unfavorable. A useful approach to anticipate such ADE risk in face of any variant is to analyze both the ADE and neutralizing epitopes of the NTD, as 
developed in Figure 3. At first glance, one can determine the balance between neutralization and facilitation and assess the risks of virus escape, $\mathrm{ADE}$ and/or both. Our molecular modeling approaches confirmed that hot mutational spots in ADE and neutralizing epitopes of the NTD give reliable information on antibody recognition of the spike protein, allowing us to determine which way the balance between neutralization and facilitation is tipping.

We recently hypothesized that the delta variant is dominating because its electrostatic surface potential of the NTD region that faces the host cell membrane has evolved to a large electropositive flat area that is complementary to the electronegative surface of lipid raft gangliosides [37]. The electrostatic potential surface value, which reflects the kinetics of virus infection, is a key parameter of a mathematic formula giving the transmissibility score (Tindex) of any SARS-CoV-2 strain. This original and straightforward approach, which has recently received experimental confirmation for both enhanced transmissibility and faster infection kinetics [55] [56], allowed us to correctly predict the rapid emergence of the delta variant $($ T-index $>10)$ over alpha $($ T-index $<4)$ even though both variants display a similar affinity for ACE-2 [37]. At present, the T-index of the delta variant is still higher than all other circulating variants (including omicron, T-index $<5$ ). Proposing a third and potentially a fourth jab to improve vaccine efficiency to face the threat of the delta variant may not be a good idea as it may further increase the amount of ADE antibodies without significant gain in neutralizing activity. Instead, we believe that it is critical to design new vaccine formulations able to induce neutralizing antibodies against this strain and, most importantly, lacking ADE epitopes in the NTD. Molecular epidemiology surveillance of SARS-CoV-2 coupled with structural analysis of variant spike proteins will certainly help to reach this goal.

\section{Transparency declaration}

- Funding. No external funding was received.

- Acknowledgements. We thank Dr. Coralie Di Scala (ORCID 0000-0003-0655-7056) and Dr. Helene Banoun (ORCID 0000-0001-8391-7989) for helpful discussions and critical advice.

- Contribution. All authors contributed equally to this study. J.F. and F.A., molecular modeling; N.Y., sequence data analysis; H.C. molecular analysis of protein-protein complexes; PG and JMS, ADE analysis of animal and human virus infections.

- Conflict of interest. The authors declare that they have no conflict of interest. 


\section{References}

1. Schmidt ME, Varga SM: The CD8 T Cell Response to Respiratory Virus Infections. Frontiers in Immunology 2018, 9.

2. Du L, Yang Y, Zhang X: Neutralizing antibodies for the prevention and treatment of COVID19. Cellular \& Molecular Immunology 2021.

3. Morens DM: Antibody-Dependent Enhancement of Infection and the Pathogenesis of Viral Disease. Clinical Infectious Diseases 1994, 19:500-512.

4. Tirado SM, Yoon KJ: Antibody-dependent enhancement of virus infection and disease. Viral Immunol 2003, 16:69-86.

5. Takada A, Feldmann H, Ksiazek TG, Kawaoka Y: Antibody-dependent enhancement of Ebola virus infection. J Virol 2003, 77:7539-7544.

6. Narayan R, Tripathi S: Intrinsic ADE: The Dark Side of Antibody Dependent Enhancement During Dengue Infection. Front Cell Infect Microbiol 2020, 10:580096.

7. Dejnirattisai W, Supasa P, Wongwiwat W, Rouvinski A, Barba-Spaeth G, Duangchinda T, Sakuntabhai A, Cao-Lormeau VM, Malasit P, Rey FA, et al: Dengue virus sero-cross-reactivity drives antibody-dependent enhancement of infection with zika virus. Nat Immunol 2016, 17:1102-1108.

8. Shukla R, Ramasamy V, Shanmugam RK, Ahuja R, Khanna N: Antibody-Dependent Enhancement: A Challenge for Developing a Safe Dengue Vaccine. Frontiers in Cellular and Infection Microbiology 2020, 10.

9. Halstead SB, Nimmannitya S, Yamarat C, Russell PK: Hemorrhagic fever in Thailand; recent knowledge regarding etiology. Jpn J Med Sci Biol 1967, 20 Suppl:96-103.

10. Halstead SB, Shotwell H, Casals J: Studies on the pathogenesis of dengue infection in monkeys. II. Clinical laboratory responses to heterologous infection. J Infect Dis 1973, 128:15-22.

11. Wang TT, Sewatanon J, Memoli MJ, Wrammert J, Bournazos S, Bhaumik SK, Pinsky BA, Chokephaibulkit K, Onlamoon N, Pattanapanyasat K, et al: IgG antibodies to dengue enhanced for FcyRIIIA binding determine disease severity. Science 2017, 355:395-398.

12. Takada A, Watanabe S, Okazaki K, Kida H, Kawaoka Y: Infectivity-enhancing antibodies to Ebola virus glycoprotein. J Virol 2001, 75:2324-2330.

13. Bardina SV, Bunduc P, Tripathi S, Duehr J, Frere JJ, Brown JA, Nachbagauer R, Foster GA, Krysztof $D$, Tortorella $D$, et al: Enhancement of Zika virus pathogenesis by preexisting antiflavivirus immunity. Science 2017, 356:175-180.

14. Marasini B, Vyas HK, Lakhashe SK, Hariraju D, Akhtar A, Ratcliffe SJ, Ruprecht RM: Mucosal AIDS virus transmission is enhanced by antiviral IgG isolated early in infection. Aids 2021.

15. Winarski KL, Tang J, Klenow L, Lee J, Coyle EM, Manischewitz J, Turner HL, Takeda K, Ward $A B$, Golding $H$, Khurana $S$ : Antibody-dependent enhancement of influenza disease promoted by increase in hemagglutinin stem flexibility and virus fusion kinetics. Proc NatI Acad Sci U S A 2019, 116:15194-15199.

16. Wen J, Cheng Y, Ling R, Dai Y, Huang B, Huang W, Zhang S, Jiang Y: Antibody-dependent enhancement of coronavirus. Int J Infect Dis 2020, 100:483-489.

17. Iwasaki A, Yang Y: The potential danger of suboptimal antibody responses in COVID-19. Nature Reviews Immunology 2020, 20:339-341.

18. Jiang S: Don't rush to deploy COVID-19 vaccines and drugs without sufficient safety guarantees. Nature 2020, 579:321.

19. Yager EJ: Antibody-dependent enhancement and COVID-19: Moving toward acquittal. Clin Immunol 2020, 217:108496.

20. van der Lubbe JEM, Rosendahl Huber SK, Vijayan A, Dekking L, van Huizen E, Vreugdenhil J, Choi Y, Baert MRM, Feddes-de Boer K, Izquierdo Gil A, et al: Ad26.COV2.S protects Syrian 
hamsters against G614 spike variant SARS-CoV-2 and does not enhance respiratory disease. npj Vaccines 2021, 6:39.

21. Clark NM, Janaka SK, Hartman W, Stramer S, Goodhue E, Weiss J, Evans DT, Connor JP: AntiSARS-CoV-2 IgG and IgA antibodies in COVID-19 convalescent plasma do not facilitate antibody-dependent enhance of viral infection. bioRxiv 2021.

22. de Alwis R, Chen S, Gan ES, Ooi EE: Impact of immune enhancement on Covid-19 polyclonal hyperimmune globulin therapy and vaccine development. EBioMedicine 2020, 55:102768.

23. Takano T, Kawakami C, Yamada S, Satoh R, Hohdatsu T: Antibody-dependent enhancement occurs upon re-infection with the identical serotype virus in feline infectious peritonitis virus infection. The Journal of veterinary medical science 2008, 70:1315-1321.

24. Vennema H, de Groot RJ, Harbour DA, Dalderup M, Gruffydd-Jones T, Horzinek MC, Spaan WJ: Early death after feline infectious peritonitis virus challenge due to recombinant vaccinia virus immunization. $J$ Virol 1990, 64:1407-1409.

25. Takano T, Tomiyama Y, Katoh Y, Nakamura M, Satoh R, Hohdatsu T: Mutation of neutralizing/antibody-dependent enhancing epitope on spike protein and $7 \mathrm{~b}$ gene of feline infectious peritonitis virus: influences of viral replication in monocytes/macrophages and virulence in cats. Virus Res 2011, 156:72-80.

26. Wang Q, Zhang L, Kuwahara K, Li L, Liu Z, Li T, Zhu H, Liu J, Xu Y, Xie J, et al: Immunodominant SARS Coronavirus Epitopes in Humans Elicited both Enhancing and Neutralizing Effects on Infection in Non-human Primates. ACS Infect Dis 2016, 2:361-376.

27. Wan Y, Shang J, Sun S, Tai W, Chen J, Geng Q, He L, Chen Y, Wu J, Shi Z, et al: Molecular Mechanism for Antibody-Dependent Enhancement of Coronavirus Entry. J Virol 2020, 94.

28. Agrawal AS, Tao X, Algaissi A, Garron T, Narayanan K, Peng BH, Couch RB, Tseng CT: Immunization with inactivated Middle East Respiratory Syndrome coronavirus vaccine leads to lung immunopathology on challenge with live virus. Hum Vaccin Immunother 2016, 12:2351-2356.

29. Liu Y, Soh WT, Kishikawa JI, Hirose M, Nakayama EE, Li S, Sasai M, Suzuki T, Tada A, Arakawa $A$, et al: An infectivity-enhancing site on the SARS-CoV-2 spike protein targeted by antibodies. Cell 2021, 184:3452-3466.e3418.

30. Li D, Edwards RJ, Manne K, Martinez DR, Schäfer A, Alam SM, Wiehe K, Lu X, Parks R, Sutherland LL, et al: In vitro and in vivo functions of SARS-CoV-2 infection-enhancing and neutralizing antibodies. Cell 2021, 184:4203-4219.e4232.

31. Yahi N, Chahinian $\mathrm{H}$, Fantini J: Infection-enhancing anti-SARS-CoV-2 antibodies recognize both the original Wuhan/D614G strain and Delta variants. A potential risk for mass vaccination? J Infect 2021.

32. Liu Y, Arase N, Kishikawa J-i, Hirose M, Li S, Tada A, Matsuoka S, Arakawa A, Akamatsu K, Ono C, et al: The SARS-CoV-2 Delta variant is poised to acquire complete resistance to wild-type spike vaccines. bioRxiv 2021:2021.2008.2022.457114.

33. Takano T, Yamada S, Doki T, Hohdatsu T: Pathogenesis of oral type I feline infectious peritonitis virus (FIPV) infection: Antibody-dependent enhancement infection of cats with type I FIPV via the oral route. J Vet Med Sci 2019, 81:911-915.

34. Fantini J, Di Scala C, Chahinian H, Yahi N: Structural and molecular modelling studies reveal a new mechanism of action of chloroquine and hydroxychloroquine against SARS-CoV-2 infection. Int J Antimicrob Agents 2020, 55:105960.

35. Fantini J, Chahinian $\mathrm{H}$, Yahi N: Synergistic antiviral effect of hydroxychloroquine and azithromycin in combination against SARS-CoV-2: What molecular dynamics studies of virus-host interactions reveal. Int J Antimicrob Agents 2020, 56:106020.

36. Fantini J, Chahinian $\mathrm{H}$, Yahi $\mathrm{N}$ : Leveraging coronavirus binding to gangliosides for innovative vaccine and therapeutic strategies against COVID-19. Biochem Biophys Res Commun 2021, 538:132-136.

37. Fantini J, Yahi N, Azzaz F, Chahinian H: Structural dynamics of SARS-CoV-2 variants: A health monitoring strategy for anticipating Covid-19 outbreaks. J Infect 2021, 83:197-206. 
38. Jo S, Kim T, Iyer VG, Im W: CHARMM-GUI: a web-based graphical user interface for CHARMM. J Comput Chem 2008, 29:1859-1865.

39. Di Scala C, Fantini J: Hybrid In Silico/In Vitro Approaches for the Identification of Functional Cholesterol-Binding Domains in Membrane Proteins. Methods Mol Biol 2017, 1583:7-19.

40. Korber B, Fischer WM, Gnanakaran S, Yoon H, Theiler J, Abfalterer W, Hengartner N, Giorgi EE, Bhattacharya T, Foley B, et al: Tracking Changes in SARS-CoV-2 Spike: Evidence that D614G Increases Infectivity of the COVID-19 Virus. Cell 2020, 182:812-827.e819.

41. Benton DJ, Wrobel AG, Xu P, Roustan C, Martin SR, Rosenthal PB, Skehel JJ, Gamblin SJ: Receptor binding and priming of the spike protein of SARS-CoV-2 for membrane fusion. Nature 2020, 588:327-330.

42. Wrapp D, Wang N, Corbett KS, Goldsmith JA, Hsieh C-L, Abiona O, Graham BS, McLellan JS: Cryo-EM structure of the 2019-nCoV spike in the prefusion conformation. Science 2020, 367:1260-1263.

43. Chi X, Yan R, Zhang J, Zhang G, Zhang Y, Hao M, Zhang Z, Fan P, Dong Y, Yang Y, et al: A neutralizing human antibody binds to the $\mathrm{N}$-terminal domain of the Spike protein of SARSCoV-2. Science 2020, 369:650-655.

44. Meng B, Kemp SA, Papa G, Datir R, Ferreira IATM, Marelli S, Harvey WT, Lytras S, Mohamed A, Gallo G, et al: Recurrent emergence of SARS-CoV-2 spike deletion H69/V70 and its role in the Alpha variant B.1.1.7. Cell Reports 2021, 35:109292.

45. Groves DC, Rowland-Jones SL, Angyal A: The D614G mutations in the SARS-CoV-2 spike protein: Implications for viral infectivity, disease severity and vaccine design. Biochemical and Biophysical Research Communications 2021, 538:104-107.

46. Kulkarni R: Antibody-Dependent Enhancement of Viral Infections. Dynamics of Immune Activation in Viral Diseases 2019:9-41.

47. Emborg H-D, Valentiner-Branth P, Schelde AB, Nielsen KF, Gram MA, Moustsen-Helms IR, Chaine $M$, Seidelin UH, Nielsen J: Vaccine effectiveness of the BNT162b2 mRNA COVID-19 vaccine against RT-PCR confirmed SARS-CoV-2 infections, hospitalisations and mortality in prioritised risk groups. medRxiv 2021:2021.2005.2027.21257583.

48. Subramanian SV, Kumar A: Increases in COVID-19 are unrelated to levels of vaccination across 68 countries and 2947 counties in the United States. European Journal of Epidemiology 2021.

49. Tetro JA: Is COVID-19 receiving ADE from other coronaviruses? Microbes Infect 2020, 22:7273.

50. Xia X: Detailed Dissection and Critical Evaluation of the Pfizer/BioNTech and Moderna mRNA Vaccines. Vaccines 2021, 9:734.

51. Zhao L, Han N, Zheng Y, Rao H, Li J, Chen Y, Yu B, Xu Y, Chen H, Gao Z, Jiang B: Detection of Antibodies Against the SARS-CoV-2 Spike Protein and Analysis of the Peripheral Blood Mononuclear Cell Transcriptomic Profile, 15 Years After Recovery From SARS. Frontiers in Cellular and Infection Microbiology 2021, 11.

52. Yang ZY, Werner HC, Kong WP, Leung K, Traggiai E, Lanzavecchia A, Nabel GJ: Evasion of antibody neutralization in emerging severe acute respiratory syndrome coronaviruses. proceedings of the national academy of sciences of the united states of america 2005, 102:797-801.

53. Pearson H: Caution raised over SARS vaccine. Nature 2005.

54. Cevik M, Grubaugh ND, Iwasaki A, Openshaw P: COVID-19 vaccines: Keeping pace with SARS-CoV-2 variants. Cell 2021.

55. Liu Y, Rocklöv J: The reproductive number of the Delta variant of SARS-CoV-2 is far higher compared to the ancestral SARS-CoV-2 virus. J Travel Med 2021, 28.

56. Zhang J, Xiao T, Cai Y, Lavine CL, Peng H, Zhu H, Anand K, Tong P, Gautam A, Mayer ML, et al: Membrane fusion and immune evasion by the spike protein of SARS-CoV-2 Delta variant. Science, 0:eabl9463. 
Figure 1. Localization of ADE epitopes on the spike protein.

A. Three distinct views of the SARS-CoV-2 spike protein (Wuhan strain). The ADE epitopes recognized by the 1052 antibody are colored in blue. The common coronavirus ADE epitope is colored in yellow, with amino acid residue D614 in yellow. The 621-640 loop that is missing in PDB: 7LAB is in green. B. ADE antibody 1052 (in cyan) bound to the monomeric spike (left panel) or to the trimeric spike protein (right panel). The N-terminal domain (NTD) and receptor-binding domain (RBD) are indicated in all models.

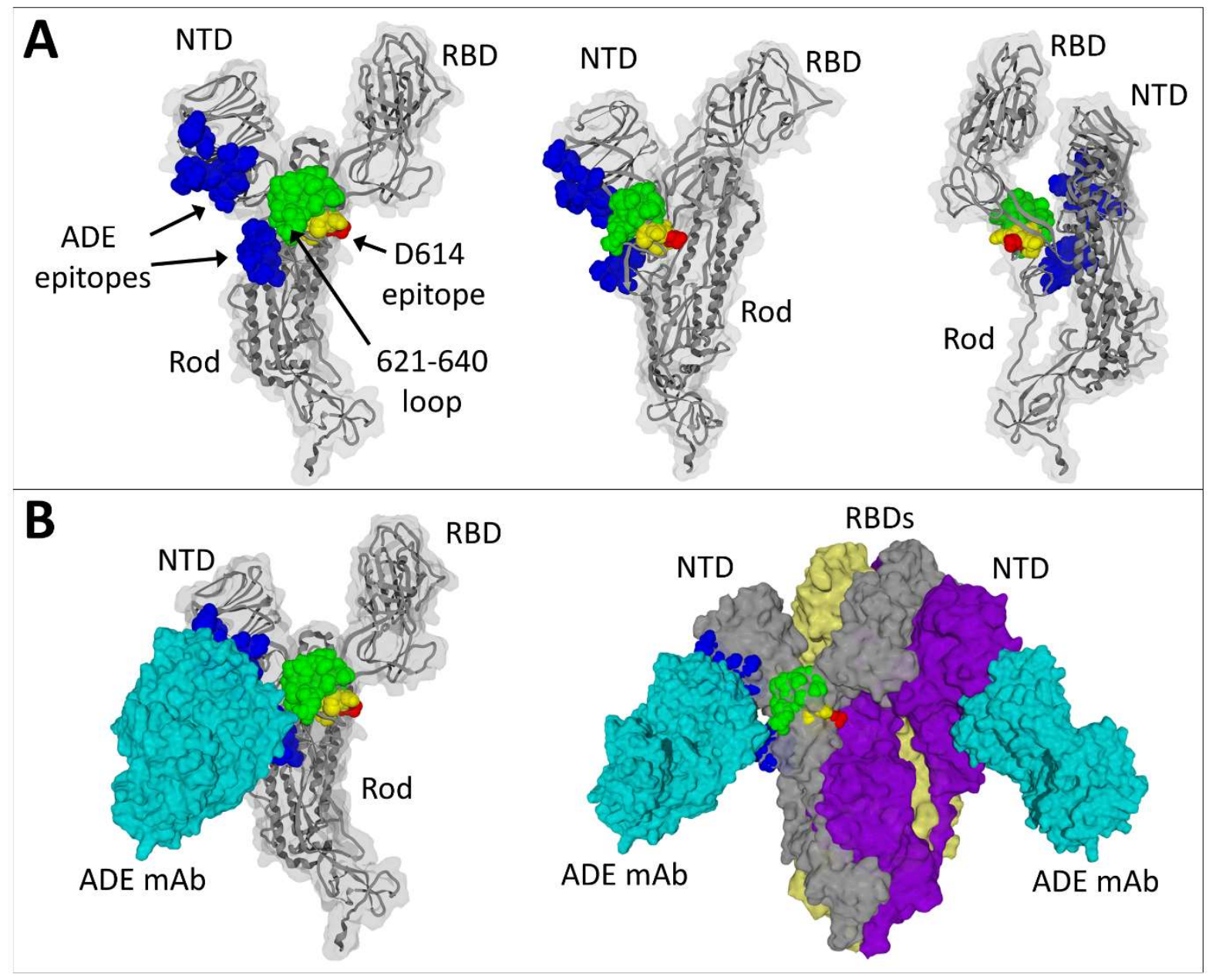


Figure 2. How the D614G mutation and the ADE antibody 1052 enhance SARS-CoV-2 infectivity.

A. Hydrogen bond between D614 (chain B) and T859 (chain C) stabilizing the trimeric spike protein (PDB: 6VSB). B. The D614G mutation renders impossible the formation of the hydrogen bond and facilitate the conformational change inducing the demasking of the RBD (PDB: 7BNM). C. Binding ADE of ADE antibody 1052 breaks the hydrogen bond between D614 and T859 (PDB: 7LAB). The arrow in panels B and C illustrates the lack of contact between vicinal spike protein monomers in the context of the trimeric association.

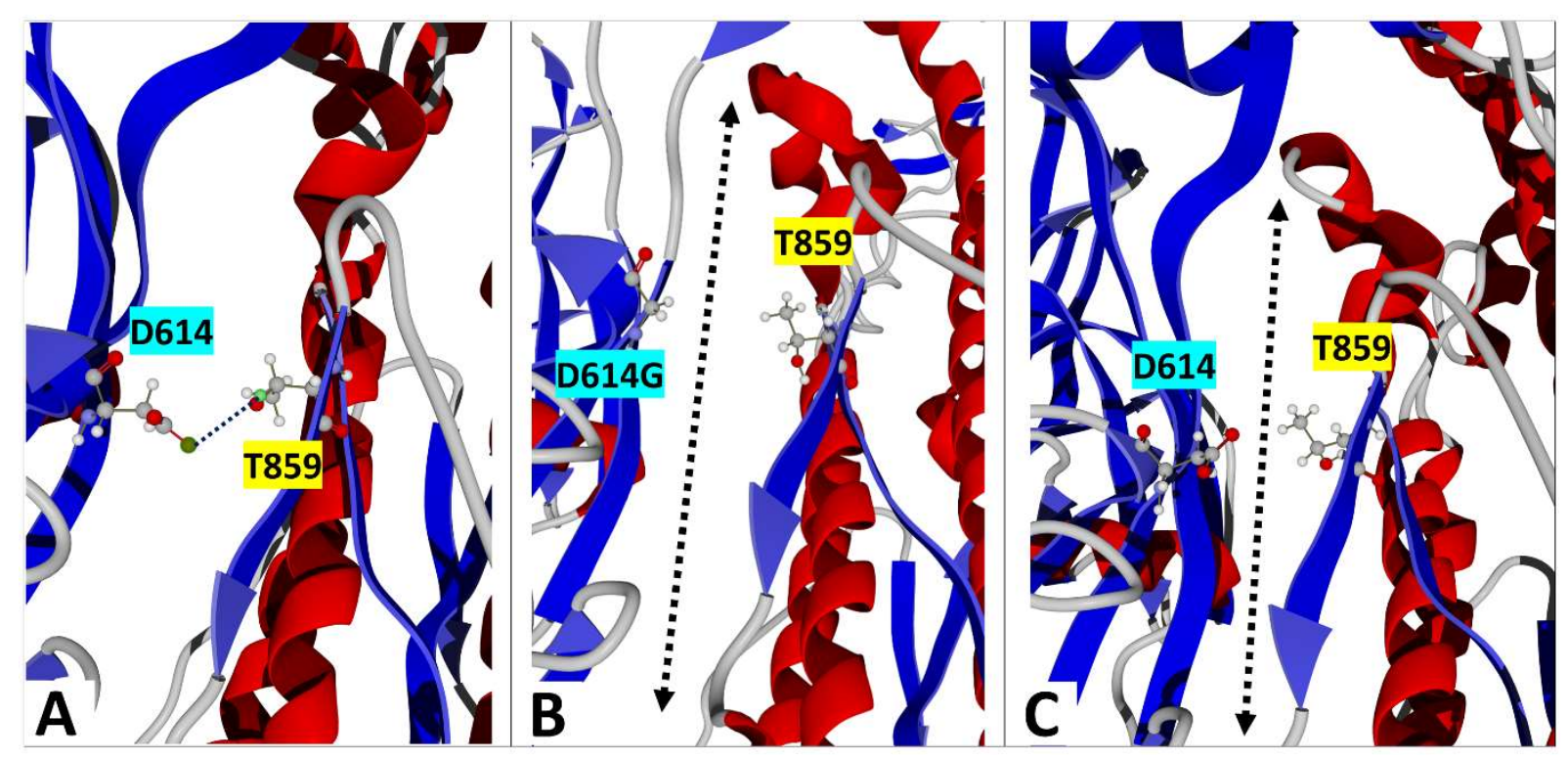


Figure 3. Amino acid sequence alignments of ADE and neutralizing epitopes in SARSCoV-2 variants.

Amino acid residue variations are highlighted in yellow. -, identity; $\Delta$, deletion. Note that the 144-158 neutralizing epitope of the $\mu$ variant displays a threonine residue ( $\mathrm{T}$, in red) inserted after Y144, then two mutations after this insertion (colored in blue). The insertion induces a shift of the amino acid sequence (highlighted in grey).

\begin{tabular}{|c|c|c|c|c|c|c|c|}
\hline & & & $\mathrm{ADE} \mathrm{mAb}$ & 1052 & & & \\
\hline & $27-32$ & $64-69$ & $211-218$ & $600-607$ & & & $689-691$ \\
\hline Wuhan Origin & AYTNSE & WFHAIH & NLVRDLPQ & PGTNTSNQ & & & SQS \\
\hline$\alpha$ B.1.1.7 UK & ------ & $-----\Delta$ & -------- & -------- & & & --- \\
\hline$\beta$ B.1.351 S Afr & ------ & ------ & $----G---$ & -------- & & & --- \\
\hline $\mathrm{n} / \mathrm{a}$ C.1.2 S_Afr & ----- & ------ & $----G---$ & ------- & & & --- \\
\hline$\gamma$ P.1 Brazil & ------ & ------ & -------- & -------- & & & --- \\
\hline$\delta$ B.1.617.2 India & ------ & ------ & -------- & -------- & & & --- \\
\hline$\mu$ B.621 Columbia & ----- & ----- & ------- & -------- & & & --- \\
\hline$\lambda$ C.37 Peru & ------ & ----- & ------- & -------- & & & --- \\
\hline o B.1.1.529 S_Afr & ------ & $---\mathrm{V}-\Delta$ & $\triangle$ IVREPEDLPQ & -------- & & & --- \\
\hline & $\mathrm{ADE} \mathrm{m}$ & b $43-3-1$ & & NEUTRA & & $\mathrm{QAB}$ & $\pm \mathrm{A} 8$ \\
\hline & & $1-617$ & & $44-158$ & & & -249 \\
\hline Wuhan Origin & & QDVNC & YYHK & NNKSWMESE & & LA & HRSYL \\
\hline$\alpha$ B.1.1.7 UK & & $-G---$ & $\Delta---$ & ------1 & & -- & ----- \\
\hline$\beta$ B.1.351 S_Afr & & $-G---$ & ---- & ------ & & & ---- \\
\hline $\mathrm{n} / \mathrm{a}$ C.1.2 S_Afr & & $-G---$ & $\Delta---$ & ------- & & & ---- \\
\hline$\gamma$ P.1 Brazil & & $-G---$ & ---- & ------1 & & & ---- \\
\hline$\delta$ B.1.617.2 India & & -G--- & ---- & $------\Delta$ & & & ---- \\
\hline p B.621 Columbia & & $-G---$ & YTSN & KNNKSWMES & & & ---- \\
\hline$\lambda$ C. 37 Peru & & $-G---$ & ---- & ------- & & & $-\mathrm{N} \Delta \Delta \Delta$ \\
\hline o B.1.1.529 S_Afr & & $-G---$ & $\Delta \Delta--$ & -------1 & & -- & ---- \\
\hline
\end{tabular}


Figure 4. Critical amino acid residues control the binding of the ADE antibody 1052 to variant spike proteins.

A. Binding of the 1052 antibody (ADE $\mathrm{mAb}$ ) to the Wuhan spike protein (PDB: 7LAB) with the NTD and RBD indicated. In the left panel, the light and heavy chains of the antibody are represented in standard secondary structures (red, $\alpha$-helix, blue, $\beta$-strand). H69 (in blue) and D215 (in yellow) are highlighted. In the right panel, a surface representation illustrates the geometric complementarity of the $\delta$ spike protein-antibody complex bound to a cluster of gangliosides GM1 figuring a lipid raft on the plasma membrane of a host cell. Note that the 1052 antibody binds simultaneously to the NTD of the spike protein and to the edge of the lipid raft. B. Molecular modeling of the NTD of several SARS-CoV-2 variants showing different levels of surface exposure of H69 (in blue) and D215 (in yellow) amino acid residues.

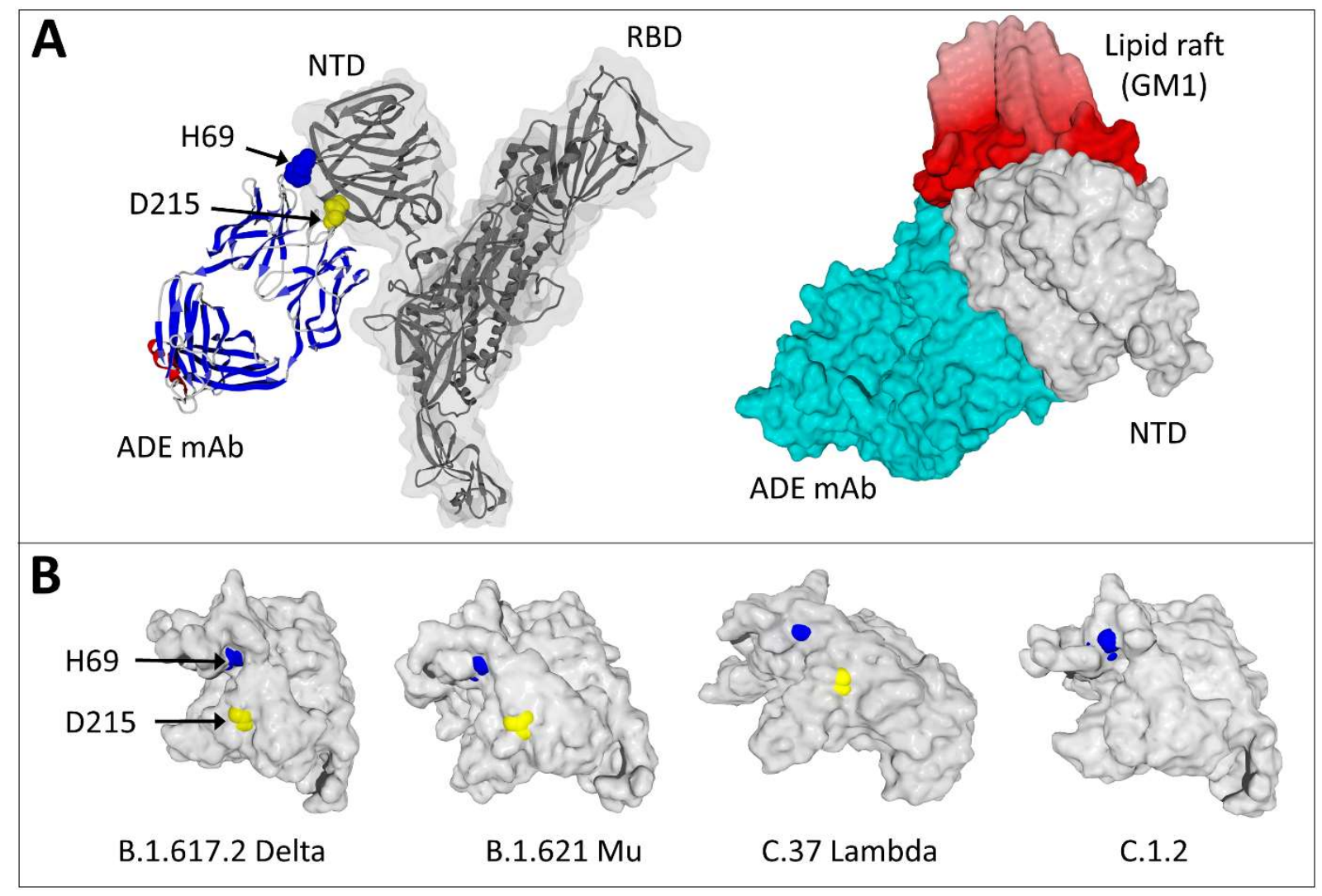


Table 1. Mutations in SARS-CoV-2 variants.

\begin{tabular}{|c|c|c|}
\hline Virus strain & NTD & Rod \\
\hline $\begin{array}{c}\text { Alpha } \alpha \\
\text { B.1.1.7 (UK) }\end{array}$ & $\frac{\Delta \mathrm{H} 69}{\underline{\Delta Y} 144}$ & $\begin{array}{l}\text { D614G P681H T716I } \\
\text { S982A D1118H }\end{array}$ \\
\hline $\begin{array}{c}\text { Beta } \beta \\
\text { B.1.351 (S_Afr) }\end{array}$ & $\begin{array}{c}\text { L18F D80A D215G } \\
\underline{\Delta L 242} \underline{\Delta \mathrm{A} 243} \underline{\Delta \mathrm{L} 244}\end{array}$ & D614G A701V \\
\hline $\begin{array}{c}n / a \\
\text { C.1.2 (S_Afr) }\end{array}$ & $\begin{array}{l}\text { P9L C136F } \Delta \text { Y144 R190S } \\
\text { D215G } \underline{\Delta 243 \underline{\Delta L 244}}\end{array}$ & $\begin{array}{c}\text { D614G H655Y N679K } \\
\text { T716I T859N }\end{array}$ \\
\hline $\begin{array}{l}\text { Gamma } \gamma \\
\text { P.1 (Brazil) }\end{array}$ & $\begin{array}{l}\text { L18F T20N P26S } \\
\text { D138Y R190S }\end{array}$ & $\begin{array}{c}\text { D614G H655Y T1027I } \\
\text { V1176F }\end{array}$ \\
\hline $\begin{array}{c}\text { Delta } \delta \\
\text { B.1.617.2 (India) }\end{array}$ & $\begin{array}{c}\text { T19R T95I G142D } \\
\underline{\Delta \mathrm{E} 156} \underline{\Delta \mathrm{F} 157} \mathrm{R} 158 \mathrm{G}\end{array}$ & D614G P681R D950N \\
\hline $\begin{array}{c}\text { Mu } \mu \\
\text { B.621 (Columbia) }\end{array}$ & T95I +143T Y144S Y145N & D614G P681H D950N \\
\hline $\begin{array}{l}\text { Lambda } \lambda \\
\text { C.37 (Peru) }\end{array}$ & $\begin{array}{c}\text { G75V T76I R246N } \Delta S 247 \\
\Delta Y 248 \Delta L 249 \underline{\Delta} 250 \\
\underline{\Delta} 251 \underline{\Delta} 252 \underline{\Delta} 253\end{array}$ & D614G T859N \\
\hline $\begin{array}{c}\text { Omicron o } \\
\text { B.1.1.529 (S_Afr) }\end{array}$ & $\begin{array}{l}\text { A67V } \frac{H 69}{\Delta V 70} \frac{\Delta 95 I ~ G 142 D}{} \\
\frac{\Delta V 143}{\underline{+2144} \frac{\Delta Y 145}{E P E}} \text { L212I }\end{array}$ & $\begin{array}{l}\text { D614G H655Y N679K } \\
\text { P681H N764K D796Y } \\
\text { N856K N954K L981F }\end{array}$ \\
\hline
\end{tabular}

Mutations patterns in the NTD and rod-like regions of the SARS-CoV-2 spike protein were obtained from the GISAID database (https://www.gisaid.org/hcov19-variants). Deletions ( $\Delta$ ) and insertions (+) are underscored. 
Table 2. Frequency of ADE and neutralizing epitope sequences.

\begin{tabular}{|c|c|c|c|c|c|c|c|c|}
\hline $\begin{array}{l}\text { Number of } \\
\text { mutations }\end{array}$ & $\begin{array}{l}1052 \mathrm{mAb} \\
\text { (NTD) } \\
\text { ADE 27-32 } \\
\text { AYTNSF }\end{array}$ & $\begin{array}{l}1052 \mathrm{mAb} \\
\text { (NTD) } \\
\text { ADE 64-69 } \\
\text { WFHAIE }\end{array}$ & $\begin{array}{c}1052 \mathrm{mAb} \\
\text { (NTD) } \\
\text { ADE 211-218 } \\
\text { NLVRDLPQ }\end{array}$ & $\begin{array}{c}1052 \mathrm{mAb} \\
\text { (Rod) } \\
\text { ADE 600-607 } \\
\text { PGTNTSN } \underline{0}\end{array}$ & $\begin{array}{l}1052 \mathrm{mAb} \\
\text { (Rod) } \\
\text { ADE 674---691 } \\
\text { YQTQ---SQS }\end{array}$ & $\begin{array}{l}\text { 43-3-14 mAb } \\
\text { (Rod) } \\
\text { ADE 611-617 } \\
\text { LYQDVNC }\end{array}$ & $\begin{array}{c}4 A 8 \mathrm{mAb} \\
\text { (NTD) } \\
\text { Neutralization 144-158 } \\
\text { YYHKNNKSWWMESEFR }\end{array}$ & $\begin{array}{c}\text { 4A8 mAb } \\
\text { (NTD) } \\
\text { Neutralization 242-249 } \\
\text { LALHRSYL }\end{array}$ \\
\hline 0 & 98.99 & 94.35 & 99.13 & 99.92 & 99.40 & $<0.05$ & $<0.05$ & 99.22 \\
\hline 1 & 0.98 & 5.46 & 0.82 & 0.05 & 0.06 & 98.70 & 3.47 & 0.36 \\
\hline 2 & - & 0.15 & $<0.05$ & - & - & 1.29 & 92.04 & 0.06 \\
\hline 3 & - & - & - & - & - & - & 4.23 & 0.09 \\
\hline 4 & - & - & - & - & - & - & $<0.05$ & 0.22 \\
\hline 5 & - & - & - & - & - & - & $<0.05$ & - \\
\hline
\end{tabular}

The frequency of mutations of each epitope sequence is calculated as the percentage of identity with the reference amino acid sequence of the SARS-CoV-2 spike protein (Wuhan strain). The most variable amino acid residues of each epitope are underlined. 1,860,489 sequences were analyzed from 2021-06-01 to 2021-11-27.

The raw data were obtained from the Los Alamos website (https://cov.lanl.gov/content/sequence/ANALYZEALIGN/analyze align.html). 